\title{
Las perspectivas de estudiantes universitarios sobre el Big Data y su papel en el sistema educativo
}

\author{
The perspectives of university students on Big Data and its role \\ in the education system
}

Cristina Negro Martínez

RECIBIDO 2/10/2019 ACEPTADO 4/8/2020 PUBLICAD0 1/12/2020

Universidad de Málaga, España

crisnegmar@uma.es

Mariano Borrego Linares

Universidad de Málaga, España

maborlin@uma.es

Antonio Matas Terrón

Universidad de Málaga, España

amatas@uma.es

\section{RESUMEN}

Hablar hoy de Big Data en educación es subrayar todas las potencialidades del manejo masivo de datos para la mejora de la calidad educativa, aunque de forma algo tardía. En este artículo se ofrecen los resultados más relevantes de una investigación realizada en un contexto universitario con estudiantes de la Universidad de Málaga. El objetivo del estudio ha sido conocer y comprender las percepciones, opiniones, conocimientos y actitudes relacionadas con el Big Data en el sistema educativo en una muestra de 104 estudiantes. La muestra del estudio fue no probabilística de carácter incidental. Para ello, se llevó a cabo un estudio de encuesta, utilizando la escala VABIDAE para recoger los datos. Se hizo un estudio psicométrico de la escala con los datos recogidos, así como un análisis estadístico descriptivo. Los resultados indican que los estudiantes tienen bastante desconocimiento sobre el Big Data y los usos que se le puede dar en el campo educativo, aunque con una percepción positiva sobre su posible impacto. Ahora bien, se muestran críticos con los beneficios y perjuicios que puede llegar a tener en la calidad educativa.

PALABRAS CLAVE Big Data, estudiantes universitarios, validación psicométrica.

\section{ABSTRACT}

Speaking about Big Data in education nowadays is to highlight the potential of massive data management for the improvement of educational quality. This article offers the most relevant results of a study conducted in a university context with students of the University of Malaga. The objective of the study was to know and understand the perceptions, opinions, knowledge and attitudes related to Big Data in the education system. For this, a survey study was conducted, using the VABIDAE scale to collect data of 104 university students. A psychometric study of the scale was done with the collected data, as 
well as a descriptive statistical analysis. The results indicate that students are quite uninformed about Big Data and its uses that can be given in the educational field, although they have a positive perception in the face data and its use. However, they are critical of the benefits and problems that can have on the quality of education.

KEYWORDS Big Data, undergraduate students, psychometric validation.

\section{INTRODUCCIÓN}

En los últimos años se ha producido un crecimiento masivo de datos originados por distintos sistemas y actividades, el ser humano ha condicionado la necesidad de cambiar, optimizar y crear métodos y modelos de almacenamiento y tratamiento de datos que solucionen los problemas que tienen las bases de datos y los sistemas de gestión de datos debido a que la situación ya no era sostenible. En esta dirección, se crea el término Big Data para reunir diferentes tecnologías, atribuidas a distintas administraciones de grandes volúmenes de datos que provienen de distintas fuentes y que se originan con gran rapidez (Li et al., 2015).

Aunque el término acuñado como Big Data se relaciona en gran medida con cantidades masivas de datos, se debe esquivar dicha perspectiva ya que Big Data no va encarrilado sólo al gran volumen, sino que abarca tanto a la variedad de datos como a la velocidad de acceso (Camargo-Vega et al., 2015). Hoy en día se ha cambiado el enfoque desde la transacción a la interacción con el objetivo de obtener mejor provecho de la información que se va creando (Mohanty et al., 2015), optimizando la utilización e interpretación de datos.

Big Data se puede definir como aquellos recursos de información caracterizados por su alto volumen, velocidad o variedad, que requieren formas de procesamiento innovadores y eficientes para la mejora del conocimiento y la toma de decisiones (Gartner, 2014). De acuerdo con esta definición, las características que definen al Big Data pueden resumirse en lo que se conoce como las tres uves: volumen, velocidad y variedad (Laney, 2001). No obstante, todos ellos suelen coincidir que el Big Data es un fenómeno que gestiona la información masiva de datos, cubriendo así su volumen, velocidad, veracidad, variedad, verificación y valor (Manyika et al., 2011; Poulovassilis, 2016).

El auge del Big Data ha propiciado que numerosas empresas vean una oportunidad para aprovecharse del tratamiento y análisis de esa masiva cantidad de datos y mejorar su producto. Esto no sólo ha provocado una revolución en el sector empresarial (IDC, 2012), sino también en el mundo de la publicidad a la hora de analizar los datos que se almacenan en las empresas de cuando circulamos por Internet, compramos a través de la web o transmitimos nuestros gustos por medio de las redes sociales. El objetivo, de empresas y publicistas, se centra en recomendarnos los productos que mejor se adecuan a nuestras preferencias (Mohanty et al., 2015).

En este aspecto entra en juego la relevancia que tiene el Big Data dentro del campo de la educación. Al igual que las empresas y la administración, la universidad viene recogiendo datos sobre los estudiantes desde hace mucho tiempo, la mayoría de ellos relacionados con su rendimiento escolar. Estos datos almacenan las calificaciones de los trabajos que el alumnado entrega, sus resultados en los exámenes, el número de convocatorias que necesitan para superar una asignatura o cuánto tiempo tardan en finalizar sus estudios (Guthrie, 2013). Analizar toda la variedad de datos que se recogen en el proceso de formación de los estudiantes se conoce como analítica del aprendizaje. Este análisis puede servir a los estudiantes 
para reflexionar sobre sus resultados y modelos de comportamiento en relación con otros compañeros; a los profesores para identificar qué estudiantes tienen más riesgo de abandonar o fracasar y necesitan más apoyo y atención; a los encargados de la calidad docente, para introducir mejoras en las asignaturas o desarrollar nuevos planes de estudio; y a los administradores, para tomar decisiones sobre temas relacionados con la promoción de los estudios, la distribución de los recursos o el proceso (Ferguson, 2014). Algunos de los impactos más destacados que el Big Data tiene en la educación son: la toma de decisiones más rápida y fundamentada; la ganancia de eficiencia; una mayor transparencia; mayor rapidez en la difusión de información; un mejor uso de las administraciones educativas; y cambios en los empleos (Cuesta, 2019).

La literatura consultada pone de relieve el escaso número de artículos centrados en el Big Data y la educación. Las investigaciones consultadas se han focalizado en el Big Data con relación a la inmersión lingüística y la educación bilingüe (González y Maroto, 2017), uso de Big Data en contextos educativos (García y Fuentes, 2016), como evaluación sobre las actitudes del profesorado en la aplicación de técnicas de Big Data (Paniagua y Cubo, 2018) y en el ámbito de los resultados docentes (Pedraza y Molina, 2017).

Los estudios adolecen de la posición que tienen los agentes implicados en la educación ante la incorporación del Big Data en dicho ámbito. En este sentido, se ha pretendido realizar un estudio del punto de vista (percepciones y opiniones), conocimientos y actitudes (oportunidades y retos) relacionadas con el Big Data en educación según los estudiantes de grado y posgrado de educación en la Universidad de Málaga. Los objetivos de esta investigación son describir la perspectiva general que estos estudiantes tienen sobre el Big Data aplicado a la educación y describir qué retos y oportunidades consideran más importantes.

Puesto que se utiliza una escala de valoración que está en proceso de validación (ver epígrafe siguiente) otro de los objetivos propuestos fue comprobar las propiedades psicométricas del instrumento con los datos de la muestra.

\section{MÉTODO}

\subsection{Participantes}

En el presente estudio participó una muestra de 104 estudiantes de la Universidad de Málaga. La muestra se construyó de forma incidental a partir de la colaboración de varios docentes que solicitaron la participación del alumnado y del grupo focal de investigadores.

La media de edad fue 22.49 (E.T.= .396), con una mediana de 22 y una desviación típica de 4.036 oscilando entre los 18 y 38 años. El $81.73 \%$ de los participantes era mujer frente al $18.3 \%$ de hombres.

\subsection{Diseño, instrumento y procedimiento}

El estudio se desarrolló siguiendo un diseño de encuesta o diseño survey a partir de un cuestionario construido ad hoc. Para ello, se utilizó la escala de Valoración del Big Data Aplicado a la Educación (VABIDAE) de Borrego et al. (2019), que se encuentra en proceso de validación. Esta escala cuenta con 31 ítems organizados en tres bloques: valoración de aspectos positivos, valoración de aspectos negativos, y emociones que induce el Big Data. Cada ítem se acompaña de una escala de apreciación con cinco opciones que varían según el bloque. Así, para los bloques de aspectos positivos y negativos la escala es: 1, No en absoluto; 2 , 
Creo que no; 3, No lo sé; 4, Creo que sí; 5, Totalmente de acuerdo. Para los ítems de emociones la escala es: 1 , Nada en absoluto; 2, Casi nada; 3, No lo sé / me resulta indiferente; 4, Algo; 5, Totalmente. En el instrumento también se completa una serie de preguntas sociodemográficas (edad, género, etc.).

Los datos se recogieron en el mes de marzo de 2019. Para ello, se solicitó la participación del profesorado de la Universidad de Málaga para la difusión del instrumento a su alumnado, siempre de forma voluntaria. El cuestionario tuvo una duración aproximada de 20 minutos y se realizó ante un/a maestro/a que resolvió las preguntas planteadas.

Para satisfacer el objetivo del estudio, y profundizar tanto en las características de la investigación como en las características métricas de la escala, se aplicó un análisis factorial exploratorio, así como un análisis de la fiabilidad a través de Alfa de Cronbach. Para los análisis se ha utilizado el paquete estadístico JASP versión 0.10 .

\section{RESULTADOS}

\subsection{Fiabilidad y Validez}

Se realizó un análisis de la consistencia interna de la escala con todos los ítems a través del procedimiento Alfa de Cronbach obteniendo una puntuación de .814. Eliminando los ítems "pérdida de privacidad del docente", "me genera aburrimiento" y “me provoca alivio” el alfa mejoraba tan sólo a .815. En función de la escasa mejora, se mantuvieron todos los ítems para el análisis de la estructura latente de los datos.

Para el análisis estructural se llevó a cabo un análisis factorial exploratorio (AFE). Inicialmente se comprobaron los supuestos básicos. El determinante de la matriz de correlación era prácticamente cero $(D=$ 1.742E-9). Por su parte, la medida de adecuación de muestreo de Keiser-Meyer-Olkin fue aceptable (.803) siendo la prueba de esfericidad de Bartlett significativa (Chi-cuadrado= 1852.127; g.l.= 465; $p<.000$ ). Esto sugiere que el análisis factorial es apropiado para estos datos.

Posteriormente se llevó a cabo el AFE por el análisis de componentes principales a través del procedimiento de extracción de máxima verosimilitud. La prueba sugirió siete factores con una varianza explicada total del 68.14\% de la extracción (Chi-cuadrado=325.265; g.l.= 269; p= .011).

Para facilitar la interpretación, se aplicó una rotación Varimax con normalización Kaiser que convergió en 7 iteraciones. En la tabla se puede ver la carga de los ítems en la matriz de factores rotados. Se ha utilizado un criterio conservador, considerado pesos inferiores a .40 como bajos (Hair et al., 2014)

Según el contenido de los ítems, se ha nombrado cada uno de los factores tal como se observa en la primera fila de la Tabla 1. En dicha tabla se muestran los pesos de cada ítem de los factores.

\subsection{Descriptivos}

El cuestionario se inicia con el bloque de oportunidades. En general, los encuestados valoran positivamente las posibilidades del Big Data. En este sentido, el 85.6\% de la muestra considera "Creo que sí" o está "Totalmente de acuerdo" con que se atienden mejor las necesidades del alumnado. Del mismo modo cuando se les preguntaba sobre la mejora de los resultados académicos existía también una opinión conjunta de un total del $74.1 \%$ de la muestra. El 76.9\% de los encuestados piensan que el Big Data puede llegar a ser de gran utilidad para personalizar la educación. 
TABLA 1. Matriz de factores rotados

\begin{tabular}{|c|c|c|c|c|c|c|c|}
\hline & $\stackrel{1}{\text { OPORTUNIDADES }}$ & $\stackrel{2}{\text { MANIPULACIÓN }}$ & $\begin{array}{c}3 \\
\text { EMOCIONES } \\
\text { NEGATIVAS }\end{array}$ & $\begin{array}{c}4 \\
\text { EMOCIONES } \\
\text { POSITIVAS }\end{array}$ & $\begin{array}{c}5 \\
\text { RETOS AL } \\
\text { CONTROL }\end{array}$ & $\begin{array}{c}6 \\
\text { PÉRDIDA DE } \\
\text { PRIVACIDAD }\end{array}$ & $\begin{array}{c}7 \\
\text { REDUCCIÓN DE } \\
\text { FUNCIONES }\end{array}$ \\
\hline $\begin{array}{l}\text { Atender mejor las necesidades } \\
\text { del alumnado }\end{array}$ & .837 & & & & & & \\
\hline Personalizar la educación & .774 & & & & & & \\
\hline $\begin{array}{l}\text { Promover la calidad educativa } \\
\text { en general }\end{array}$ & .748 & & & & & & \\
\hline $\begin{array}{l}\text { Producir recursos educativos } \\
\text { adaptados al alumnado }\end{array}$ & .710 & & & & & & \\
\hline $\begin{array}{l}\text { Mejorar los resultados acadé- } \\
\text { micos }\end{array}$ & .686 & & & & & & \\
\hline $\begin{array}{l}\text { Ayuda a prevenir el fracaso } \\
\text { escolar }\end{array}$ & .650 & & & & & & \\
\hline $\begin{array}{l}\text { Mejorar la organización } \\
\text { de los centros escolares }\end{array}$ & .565 & & & & & & \\
\hline Aumento del poder político & & .838 & & & & & \\
\hline $\begin{array}{l}\text { Control del sistema educativo } \\
\text { por parte de empresas }\end{array}$ & & .821 & & & & & \\
\hline $\begin{array}{l}\text { Control del sistema educativo } \\
\text { or parte de gobiernos }\end{array}$ & & .770 & & & & & \\
\hline Manipulación del sistema & & .697 & & & & & \\
\hline $\begin{array}{l}\text { Aumento del poder de gestores } \\
\text { de centros }\end{array}$ & & .622 & & & & & \\
\hline Ataques informáticos & & .512 & & & & & \\
\hline Siento vergüenza & & & .860 & & & & \\
\hline Siento culpa & & & .822 & & & & \\
\hline Me siento impotente & & & .687 & & & & \\
\hline Me provoca ansiedad & & & .633 & & & & \\
\hline Me hace sentir enojado & & & .602 & & & & \\
\hline \multicolumn{8}{|l|}{ Me genera aburrimiento } \\
\hline Me provoca esperanza & & & & .735 & & & \\
\hline Me hace sentir orgulloso & & & & .732 & & & \\
\hline Me provoca alivio & & & & .653 & & & \\
\hline El tema me divierte & & & & .495 & & & \\
\hline $\begin{array}{l}\text { Mejorar la selección de los } \\
\text { docentes }\end{array}$ & & & & & .719 & & \\
\hline Evitar el plagio & & & & & .477 & & \\
\hline Mejorar la empleabilidad & .418 & & & & .467 & & \\
\hline $\begin{array}{l}\text { Facilitar la toma de decisiones } \\
\text { a nivel político }\end{array}$ & & & & & .408 & & \\
\hline $\begin{array}{l}\text { Pérdida de privacidad del alum- } \\
\text { nado }\end{array}$ & & & & & & .705 & \\
\hline $\begin{array}{l}\text { Pérdida de privacidad del } \\
\text { docente }\end{array}$ & & & & & & .686 & \\
\hline Pérdida de funciones del docente & & .462 & & & & & .630 \\
\hline $\begin{array}{l}\text { Pérdida de la socialización propia } \\
\text { de la escuela }\end{array}$ & & .466 & & & & & .478 \\
\hline
\end{tabular}


En referencia a la opción de si mejorará la empleabilidad, hubo más disparidad en los resultados, donde sólo el $17.3 \%$ estaba “Totalmente de acuerdo". Asimismo, el $28.8 \%$ cree que sí puede llegar a ser de utilidad. El porcentaje mayoritario, con un 37.5\%, corresponde a la respuesta de "No lo sé".

En el ítem sobre evitar el plagio podemos observar que los participantes no están seguros sobre su respuesta debido a que el $33.7 \%$ contestó “No lo sé” y el $30.8 \%$ respondió “Creo que sí”. Por tanto, observamos que los participantes no tienen mucho conocimiento sobre qué usos puede tener el Big Data en este campo.

En relación a la opción de mejorar la organización de los centros escolares, podemos encontrar controversia en los resultados. Un $48.1 \%$ cree que el Big Data es de gran utilidad para ello. Sin embargo, podemos observar que un $17.3 \%$ respondió "No lo sé" y un 19.2\% está "Totalmente de acuerdo".

Entre las respuestas sobre la mejora de la selección docente se observan diferencias bastante significativas. El $29.8 \%$ de los participantes se posiciona indeciso. Del mismo modo, con el mismo porcentaje, podemos encontrar que hay personas que creen que sí es de gran utilidad. En el extremo contrario podemos observar que tan sólo un $8.7 \%$ está “Totalmente en desacuerdo", mientras que un 13.5\% afirma que está "Totalmente de acuerdo" con la mejora de la selección.

La opción sobre producir recursos educativos adaptados al alumnado presenta una opción mayoritaria entre "Creo que sí" y “Totalmente de acuerdo", sumando un 80.8\%. De acuerdo con los datos de este estudio, a la hora de preguntar sobre la facilitación de la toma de decisiones a nivel político, podemos ver que el $34.6 \%$ no sabe realmente si el Big Data es útil para esto. Hay porcentajes con diferencias no significativas en el resto de los ítems, siendo una de las preguntas con menos consenso.

Los participantes de esta muestra han elegido en su mayoría la opción "Creo que sí" (37.5\%) ante la pregunta de si promueve la calidad educativa en general. En cambio, podemos percibir que existe disparidad debido a que el 20.2\% respondió "Creo que no" y el 26\% estaba "Totalmente de acuerdo".

Por último, podemos destacar que el 64.4\% afirma que el Big Data ayudaba de forma positiva a evitar el fracaso escolar.

En relación con la edad y el sexo de los participantes, no se han encontrado diferencias significativas en las respuestas, por tanto, podemos concluir que no es un factor influyente en la investigación.En relación a los posibles problemas o retos asociados al Big Data, los participantes se manifiestan indecisos ante el ítem "Pérdida de privacidad del alumnado". Obtenemos opiniones muy heterogéneas que marcan una clara diferencia de dos grupos, siendo la opción mayoritaria “Creo que sí” con un 40.4\% de las respuestas. Por el contrario, el porcentaje más bajo corresponde a la respuesta "No en absoluto" con un 5.8\%.

Los resultados correspondientes a "Pérdidas de privacidad del docente" se caracterizan por ser en su mayoría homogéneos, con porcentajes que rondan entre el 27.9\%, quienes eligen la opción "Creo que no", un $22.1 \%$ que se encuentra indeciso, y un $26.9 \%$ opta por "Creo que sí".

Con respecto a la opción de "Pérdida de la socialización propia de la escuela", podemos analizar que, en su mayoría, los estudiantes se han manifestado de acuerdo con este ítem. Siendo un 33.7\% los que han contestado "Creo que sí", y un 31.7\% los estudiantes que están “Totalmente de acuerdo".

De entre las diferentes opciones, podemos comprobar que, en "Ataques informáticos”, la mayoría de los estudiantes se han posicionado a favor con un 32.7\%. Sin embargo, solo el 3.8\% manifiesta desacuerdo al responder "No en absoluto".

Entre las respuestas con relación al ítem "Pérdidas de funciones del docente", podemos observar cierto equilibrio entre los porcentajes. A excepción de una minoría, concretamente el 8.7\%, el resto manifiesta que está en total desacuerdo. 
TABLA 2. Porcentajes de respuesta para el bloque de oportunidades

\begin{tabular}{|c|c|c|c|c|c|}
\hline İTEM & 1 NO EN ABSOLUTO & 2 CREO QUE NO & 3 NO LO SÉ & 4 CREO QUE Sí & 5 TOTALMENTE DE ACUERDO \\
\hline Atender mejor las & & & & & \\
\hline $\begin{array}{l}\text { necesidades del } \\
\text { alumnado }\end{array}$ & 2.8 & 5.8 & 5.8 & 50 & 35.6 \\
\hline $\begin{array}{l}\text { Mejorar los resultados } \\
\text { académicos }\end{array}$ & 3.8 & 4.8 & 17.3 & 48.1 & 26 \\
\hline $\begin{array}{l}\text { Personalizar la } \\
\text { educación }\end{array}$ & 5.8 & 6.7 & 10.6 & 42.3 & 34.6 \\
\hline $\begin{array}{l}\text { Mejorar la } \\
\text { empleabilidad }\end{array}$ & 4.8 & 11.6 & 37.5 & 28.8 & 17.3 \\
\hline Evitar el plagio & 5.8 & 12.5 & 33.6 & 30.8 & 17.3 \\
\hline $\begin{array}{l}\text { Mejorar la organiza- } \\
\text { ción de los centros } \\
\text { escolares }\end{array}$ & 3.9 & 11.5 & 17.3 & 48.1 & 19.2 \\
\hline $\begin{array}{l}\text { Mejorar la selección } \\
\text { de los docentes }\end{array}$ & 8.6 & 18.3 & 29.8 & 29.8 & 13.5 \\
\hline $\begin{array}{l}\text { Producir recursos } \\
\text { educativos adaptados } \\
\text { al alumnado }\end{array}$ & 2.8 & 5.8 & 10.6 & 43.3 & 37.5 \\
\hline $\begin{array}{l}\text { Facilitar la toma de } \\
\text { decisiones a nivel } \\
\text { político }\end{array}$ & 12.5 & 23.1 & 34.6 & 19.2 & 10.6 \\
\hline $\begin{array}{l}\text { Promover la calidad } \\
\text { educativa en general }\end{array}$ & 2.9 & 13.5 & 20.1 & 37.5 & 26 \\
\hline $\begin{array}{l}\text { Ayuda a prevenir el } \\
\text { fracaso escolar }\end{array}$ & 8.7 & 12.5 & 14.4 & 31.7 & 32.7 \\
\hline
\end{tabular}

Con respecto al "Aumento de poder de gestores de centro", encontramos en nuestro análisis una clara predominancia de respuesta con un $46.2 \%$ que dicen no saberlo. Por otro lado, se registra un porcentaje del $5.8 \%$ de respuestas que escogen la opción "No en absoluto".

De entre las respuestas obtenidas en el apartado "Aumento de poder de político", podemos comprobar que los porcentajes se encuentran bastante igualados en lo que respecta a respuestas como "No lo sé" (30.8\%), "Creo que sí" (28.8\%) y “Totalmente de acuerdo" (23.1\%). Dichos porcentajes presentan una clara diferencia con el número de respuestas que dicen "No en absoluto", con un $6.7 \%$.

En relación con el ítem "Manipulación del sistema", la mayoría de las respuestas con un 41.3\% señalan que creen tener conocimiento sobre el tema. Siendo la minoría, con un 5.8\%, el número de respuestas que aseguran no conocer nada del tema.

En correspondencia con la opción "Control del sistema educativo por parte del gobierno", hemos podido analizar claras diferencias en las respuestas obtenidas. Por una parte, vemos que el $31.8 \%$ niega tener conocimientos sobre dicho ítem. Por otro lado, podemos apreciar que la mayoría de las respuestas, con un $68.2 \%$, presentan connotaciones positivas, estando de acuerdo. 
Por último, se debe indicar que no se han encontrado diferencias significativas, con respecto al "Control del sistema educativo por parte de empresas", en las respuestas que se posicionan positivamente sobre dicha opción, con un 30.8\% para ambas respuestas (Creo que sí y Totalmente de acuerdo). Encontrando, por el contrario, una clara diferencia porcentual del 3.8\% que indican que no están de acuerdo con ello.

TABLA 3. Porcentajes de respuesta para el bloque de retos

\begin{tabular}{|c|c|c|c|c|c|}
\hline ÍTEM & 1 NO EN ABSOLUTO & 2 CREO QUE NO & 3 NO LO SÉ & 4 CREO QUE SÍ & 5 TOTALMENTE DE ACUERDO \\
\hline $\begin{array}{l}\text { Pérdida de privacidad } \\
\text { del alumnado }\end{array}$ & 5.8 & 15.4 & 8.6 & 40.4 & 29.8 \\
\hline $\begin{array}{l}\text { Pérdida de privacidad } \\
\text { del docente }\end{array}$ & 7.7 & 27.9 & 22.1 & 26.9 & 15.4 \\
\hline $\begin{array}{l}\text { Pérdida de la socia- } \\
\text { lización propia de la } \\
\text { escuela }\end{array}$ & 4.8 & 14.4 & 15.4 & 33.7 & 31.7 \\
\hline Ataques informáticos & 3.8 & 15.4 & 20.2 & 32.7 & 27.9 \\
\hline $\begin{array}{l}\text { Pérdida de funciones } \\
\text { del docente }\end{array}$ & 8.6 & 23.1 & 15.4 & 29.8 & 23.1 \\
\hline $\begin{array}{l}\text { Aumento del poder de } \\
\text { gestores de centro }\end{array}$ & 5.8 & 6.7 & 46.2 & 25 & 16.3 \\
\hline $\begin{array}{l}\text { Aumento del poder de } \\
\text { políticos }\end{array}$ & 6.7 & 10.6 & 30.8 & 28.8 & 23.1 \\
\hline $\begin{array}{l}\text { Manipulación del } \\
\text { sistema }\end{array}$ & 5.8 & 6.7 & 12.5 & 41.3 & 33.7 \\
\hline $\begin{array}{l}\text { Control del sistema } \\
\text { educativo por parte de } \\
\text { gobiernos }\end{array}$ & 3.9 & 13.5 & 14.4 & 36.5 & 31.7 \\
\hline $\begin{array}{l}\text { Control del sistema } \\
\text { educativo por parte de } \\
\text { empresas }\end{array}$ & 3.8 & 12.5 & 22.1 & 30.8 & 30.8 \\
\hline
\end{tabular}

Con relación a las distintas emociones que provoca el tema del Big Data en educación, se encuentran datos muy interesantes por parte de los participantes.

El primer ítem, el cual hace referencia a la diversión que despierta el Big Data, muestra como el 55.8\% está "Totalmente de acuerdo" y "Algo de acuerdo" con dicha afirmación. En cambio, el 27.9\% se muestra "Indiferente" o no le provoca emoción alguna.

Asimismo, el ítem "Me provoca esperanza" registra bastante disparidad en los resultados. Por un lado, el $44.3 \%$ le resulta indiferente o no le genera casi ninguna esperanza. Por otro lado, el $48.1 \%$ está "algo de acuerdo" o "Totalmente de acuerdo" con que el Big Data le provoca esperanza.

Con relación a sí le hacían "Sentir orgulloso", no se encontraron diferencias significativas entre los encuestados. El 61.6\% coincide en que le resulta indiferente o le interesa algo.

El "sentirse enojado" no atrae los sentimientos de la mayoría de los encuestados y se muestra entre nada y casi nada el 52.9\% de los participantes, mostrándose algo enojados tan sólo el 2.9\%. 
El ítem “Me provoca ansiedad" se inclina hacia los ítems negativos e indiferentes (80.7\%). Esto nos muestra que las personas, en general, tienen una buena visión hacia el Big Data y su relación con la educación.

La respuesta "Nada en absoluto" obtuvo el 63.5\% con relación a la pregunta de si el Big Data les "Provocaba vergüenza".

La opción siento culpa, es el ítem que muestra mayor conformidad, con un 63.5\%, hacia la respuesta "Nada en absoluto".

Respecto al sentimiento de alivio, tampoco se hallaron diferencias significativas. La opción mayoritaria en todos los ítems fue "Indiferente" y "Algo de acuerdo" (54.9\%). Aunque también una gran parte de los participantes (27.9\%) se decantaron por la opción "Nada en absoluto”.

Centrándonos en la opción "Me siento impotente", constatamos que el 44.2\% respondieron estar "Nada en absoluto de acuerdo", mientras que el menor porcentaje, con un 3.8\%, corresponde a la opción "Totalmente de acuerdo".

En el ítem "Me genera aburrimiento" se comprobó que las opciones más elegidas entre todos los participantes corresponden, una vez más, a las respuestas con connotación negativa, sumando en su totalidad el $80.8 \%$.

TABLA 4. Porcentajes de respuesta para el bloque de emociones

\begin{tabular}{|c|c|c|c|c|c|}
\hline ÍTEM & 1 NADA EN ABSOLUTO & 2 CASI NADA & $\begin{array}{l}\text { 3 NO LO SÉ / ME RESULTA } \\
\text { INDIFERENTE }\end{array}$ & 4 ALGO & 5 TOTALMENTE \\
\hline El tema me divierte & 7.7 & 8.6 & 27.9 & 43.3 & 12.5 \\
\hline $\begin{array}{l}\text { Me provoca } \\
\text { esperanza }\end{array}$ & 7.7 & 21.1 & 23.1 & 35.6 & 12.5 \\
\hline $\begin{array}{l}\text { Me hace sentir } \\
\text { orgulloso }\end{array}$ & 13.5 & 18.3 & 40.4 & 21.1 & 6.7 \\
\hline $\begin{array}{l}\text { Me hace sentir } \\
\text { enojado }\end{array}$ & 33.7 & 19.2 & 24 & 20.2 & 2.9 \\
\hline $\begin{array}{l}\text { Me provoca } \\
\text { ansiedad }\end{array}$ & 39.4 & 17.3 & 24 & 14.4 & 4.9 \\
\hline Siento vergüenza & 52.9 & 17.3 & 24 & 4.8 & 1 \\
\hline Siento culpa & 63.4 & 13.5 & 19.2 & 2.9 & 1 \\
\hline Me provoca alivio & 27.9 & 14.4 & 33.7 & 21.1 & 2.9 \\
\hline Me siento impotente & 44.2 & 14.4 & 26.9 & 10.6 & 3.9 \\
\hline $\begin{array}{l}\text { Me genera } \\
\text { aburrimiento }\end{array}$ & 29.8 & 23.1 & 27.9 & 17.3 & 1.9 \\
\hline
\end{tabular}

Por último, los participantes contestaron a la pregunta “¿Usaría el Big Data en su profesión si fuera posible? Con una escala numérica de 1 (No en absoluto) a 5 (Sí, por supuesto). La mayoría eligieron los valores 3 y $4(66.3 \%)$ con una media de 3.087 puntos (E.M.= .101) y una desviación típica de 1.034 puntos. 
FIGURA 1. Histograma del factor oportunidades

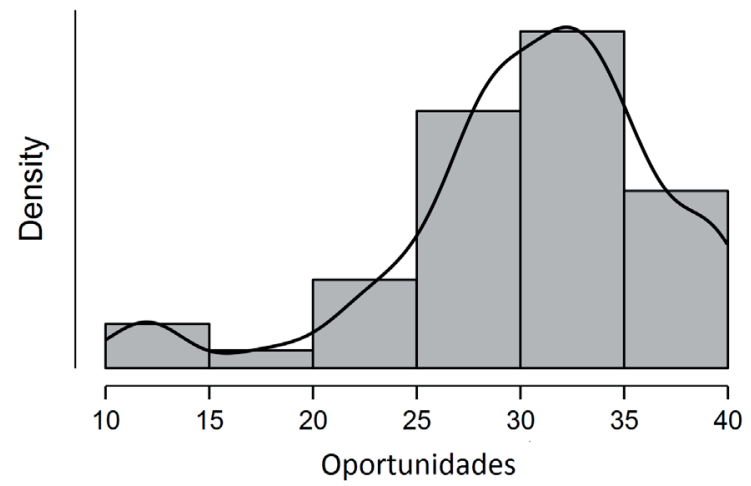

FIGURA 2. Histograma del factor manipulación

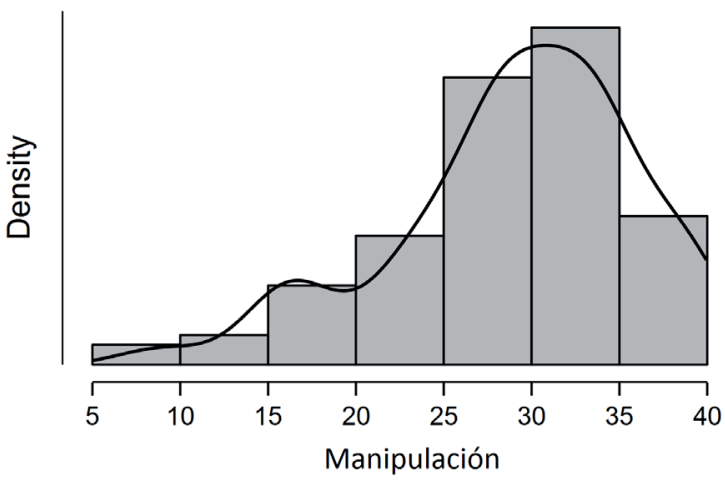

FIGURA 3. Histograma del factor de retos de control

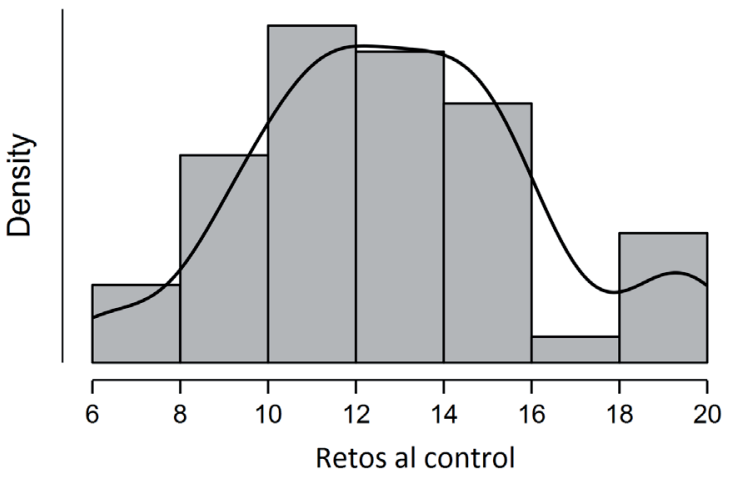

FIGURA 4. Histograma del factor emociones positivas

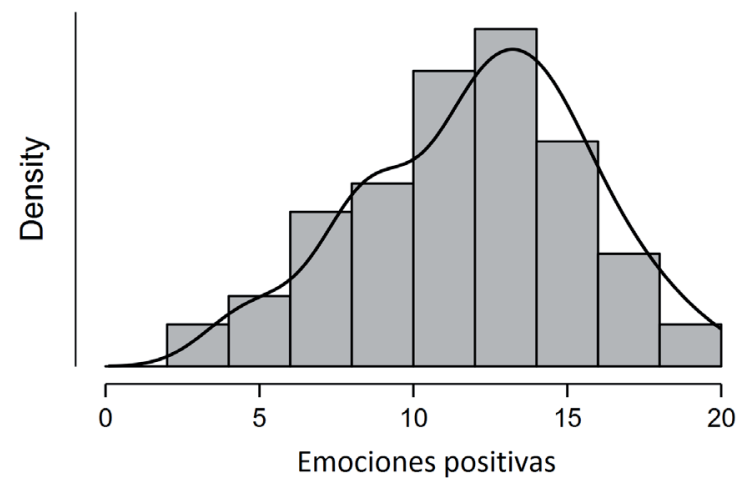

Finalmente, se ha realizado un análisis descriptivo de las puntuaciones generadas en AFE. Para ello, se calcularon los promedios en cada factor a partir de las puntuaciones de los ítems que los componen.

Así, la media del factor de oportunidades es de 30.442 (E.S.= .633) con una desviación típica de 6.453. Por su parte, la manipulación presenta un promedio de 29.058 (E.S.= .683) y una desviación típica de 6.963, mientras que retos al control tiene un promedio de 12.971 (E.S.= .318) y una desviación típica de 3.242. Esta última, presenta mayor heterogeneidad que las oportunidades y la manipulación (figura 3). En la figura 1 y 2 se puede observar que, además, los factores de oportunidades y manipulación están sesgados negativamente.

En relación con las emociones, los positivos tienen una media de 12.144 (E.S.= .360) y una desviación típica de 3.669, frente a las emociones negativas que tiene una media de 10.308 (E.S.= .447) y una desviación típica de 4.563. Ambos factores presentan distribuciones con un relativo alto sesgo, siendo .773 para las emociones positivas y de .812 para las emociones negativas, tal y como se aprecia en la figura 4 y en la figura 5.

Se ha obviado la presentación de los factores sexto y séptimo puesto que coinciden con lo reflejado en el bloque de retos que se ha comentado anteriormente (tabla 3).

\section{DISCUSIÓN}

En primer lugar, hay que destacar que el instrumento utilizado presenta un buen ajuste, tanto en la estructura teórica en la que se basa, como en la naturaleza de los datos. Por lo anterior, la escala utilizada podría presentarse como un instrumento que podría ser estandarizado. Así se podría impulsar una proyección más potente en términos pedagógicos.

Además, debemos subrayar que la escala llega a explicar un alto porcentaje de la varianza, apoyando aún más su validez y utilidad, según los criterios que marcan autores con Hair et al. (2014). 
FIGURA 5. Histograma del factor emociones negativas

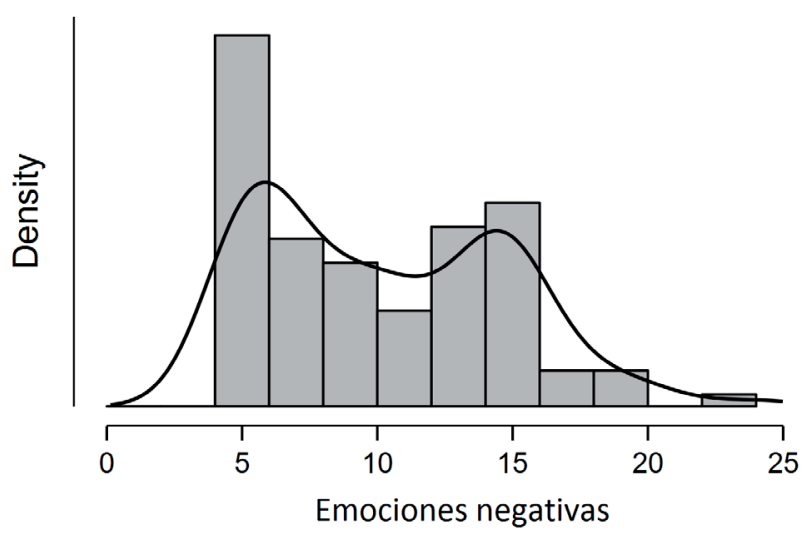

En estudios posteriores, se podría tratar de generalizar esta escala como instrumento para evaluar otras experiencias de las personas con las tecnologías e innovaciones tecnológicas, no sólo con el Big Data. Esto se concibe como una oportunidad de traslación y transferencia de aprendizajes.

En relación con los resultados obtenidos, las percepciones, opiniones, conocimientos, oportunidades y retos que ofrecen el Big Data en la educación son muy importantes, debiendo ser conocidas y comprendidas por todas las partes. Esto se debe

a que tienen un papel y ejercen una influencia en la implementación del Big Data en el sistema educativo, especialmente en la mejora del desempeño de una organización pública o privada (Yudatama et al., 2017).

Los mecanismos de engranaje que interactúan en el sistema educativo son complicados, pues está influenciado tanto por factores fisiológicos como por factores cognitivos y sociales. La tecnología del Big Data puede llevar a cabo con eficacia análisis de datos, utilizándose ampliamente en ámbitos sociales en los últimos años. El Big Data puede ser una herramienta potencial que proporcione a los estudiantes cierto nivel de control sobre el análisis de aprendizajes como un medio para aumentar el aprendizaje autorregulado y el rendimiento académico (Roberts et al., 2017).

El Big Data en educación ha llevado a investigadores y desarrolladores a ver las posibilidades de cómo introducir diferentes tecnologías para procesar y generar información, para apoyar el aprendizaje de los estudiantes. Asimismo, hay un gran interés en este campo de investigación y su aplicación, en el Sistema Educativo, cambiará su mentalidad pedagógica, además de la de los propios centros de formación (Matas et al., 2020). A pesar de la creciente investigación sobre Big Data en educación y su aparente valor para el aprendizaje, muchos centros educativos se están retrasando en la implementación de proyectos con Big Data (Macfadyen, 2017). Esta investigación ha reflejado que los participantes tienen un desconocimiento prácticamente total del Big Data. Este desconocimiento también implica una falta de elementos para la reflexión y concepción crítica de sus potencialidades y complejidad desde un punto de vista holístico. Por ello, para que el Big Data pueda ser una realidad en la educación, es necesario una formación previa, tanto del profesorado como del alumnado, para hacer un uso correcto de esta herramienta. Prueba de ello, se demuestra en la investigación realizada por Naujokaitienè et al. (2020) al confirmar que los profesores universitarios, a pesar de ser conscientes de la posibilidad de acceder al Big Data y utilizarlos para el diseño del plan de estudios y la participación del alumnado, tienen una comprensión muy limitada de cómo estos datos podrían ayudarles a realizar cambios en el diseño del plan de estudios. Eynon (2013) nos advirtió sobre el uso excesivo del Big Data en la educación como una forma de "arreglo técnico", en lugar de como una forma de empoderar a los investigadores para que lleven a cabo una mejor investigación educativa.

A medida que los grandes datos en la educación se convierten en un paradigma de investigación general, es necesario abordar las cuestiones de conceptualización antes de que sean ampliamente aceptados. Se necesita una nueva concepción de los grandes datos en el contexto de la investigación educativa, que tengan en cuenta la complejidad de los entornos educativos y la naturaleza de los datos que se recopilan. El Big Data crea oportunidades únicas para la investigación. Sin embargo, estas oportunidades no son accesibles inmediatamente a todos los investigadores de la educación, a menos que se ofrezcan oportunidades 
de desarrollo profesional (Daniel, 2017). Además, el establecimiento de programas de investigación educativa que utilicen Big Data requerirá que se aborden cuestiones de epistemología, ontología, metodología y desigualdad para aprovechar los resultados de Big Data en educación. La valoración general de los participantes del Big Data es bastante positiva, viéndolo como una oportunidad para la mejora del proceso de aprendizaje, haciendo especial hincapié en la utilidad para atender la diversidad del aula, permitiendo personalizar aún más la educación y mejorando los resultados académicos.

Por otro lado, es importante abordar las cuestiones de infraestructura, herramientas y capacidad humana necesarias para la recopilación, limpieza, análisis y distribución eficiente de grandes conjuntos de datos. Además, las preocupaciones críticas sobre la privacidad, la ética y el acceso siguen siendo una cuestión importante (Gasevic et al., 2016). A medida que las instituciones aumenten la necesidad de compartir datos educativos, será imperativo que se elaboren normas nacionales e internacionales sobre cuestiones críticas para la investigación en materia de educación, a fin de abordar los factores de seguridad e interoperabilidad de los datos, privacidad y acceso. Los educadores pueden participar en la recolección de diversas formas de datos para la mejora de la clase, en lugar de para la investigación (Ho, 2017), y es posible que la reutilización de estos datos para la investigación pueda no ser ética. En este sentido, los participantes minimizan los posibles aspectos negativos que puede tener el Big Data. Esto puede deberse a una falta de reflexión sobre las consecuencias que puede tener el uso de esta tecnología y cómo podría suponer un problema para el avance educativo si no se utiliza correctamente. Esta falta de conocimiento también se ve reflejado en el bloque de las emociones, donde se observa cómo los participantes tienen mayoritariamente sensaciones positivas y escasamente negativas.

Tras haber pasado el cuestionario podemos ver que los datos de esta muestra coinciden con las investigaciones que algunos autores han realizado sobre el Big Data. A continuación, comentamos los datos más destacados que creemos merecen especial interés.

Según De la Fuente y Quijano (2018), la evolución del Big Data ha ocasionado que se vean afectados aspectos como la privacidad y la seguridad de los datos que navegan por la web. Hecho que coincide con los resultados obtenidos en nuestra investigación, la cual en su mayoría se posicionan a favor de que los datos manejados por el Big Data no se encuentran totalmente protegidos, manifestándolo cuando se les cuestiona sobre la pérdida de privacidad, tanto del alumnado como del docente. Sin embargo, dentro del campo educativo, se puede ver como una herramienta, dado que las aplicaciones de educación recopilan una cantidad asombrosa de datos (Big Data) del profesorado y estudiantes, los cuales se convierten en algoritmos que dan forma al aprendizaje del alumnado (Sancho-Gil, 2020).

Por todo ello, los autores anteriormente mencionados afirman que se ha desatado el miedo pues, hasta el momento, el Reglamento General de Protección de Datos no ha exigido nada al respecto, entendiendo así que los datos estarán expuestos a cualquier persona, incluidas aquellas que quieran hacer un uso malintencionado de los mismos.

Por otro lado, los datos expuestos por el Parlamento Europeo sobre el Big Data con relación al crecimiento económico y de empleo en Europa, muestran que este fenómeno ha dado lugar a un aumento de 3.75 millones de nuevos puestos de trabajo (Gómez, 2016). Este dato contrasta con un gran número de respuestas recogidas en nuestra investigación, las cuales han negado la posibilidad de que mejore el empleo gracias al Big Data, aunque se ha de resaltar que otra gran cantidad de respuestas coinciden con los datos ofrecidos por el Parlamento Europeo.

Otro dato importante que destacar de nuestra investigación ha sido el alto porcentaje de resultados 
que afirman que el Big Data produce recursos adaptados al alumnado, un dato que contrasta con Domínguez (2018), quien afirma en su estudio que los grandes almacenamientos de datos no dan respuestas inmediatas a las cuestiones más buscadas en internet. Sin embargo, este mismo autor aboga por incrementar mayores diseños de investigación a través de los cuales se ponga más atención a aquellas funciones que mejoren el aprendizaje de los estudiantes, apostando por nuevos instrumentos que estructuren y ayuden a seleccionar aquella información que el estudiante desea conseguir de forma virtual.

\section{CONCLUSIONES}

Esta investigación concluye que es necesario que los futuros estudios se centren en explorar los temas abordados anteriormente, así como reconocer estrategias para apoyar las investigaciones educativas. El Big Data, en muy poco tiempo, ha pasado de ser una tecnología nueva a una realidad cada vez más presentes en todos los campos de la sociedad, incidiendo con especial fuerza en el sistema educativo. Pasar de una recopilación de datos aséptica o neutral a una recopilación masiva con intencionalidad positiva e integradora para la mejora de la calidad educativa, no es sólo necesario en términos de dicho control de calidad, sino también se trata de un compromiso moral y ético.

Aunque esta actualización pedagógica no se puede llevar a cabo sin desarrollar antes una formación correcta del profesorado en esta materia. El éxito del Big Data en la educación dependerá en gran medida de la capacidad que tengan los profesionales de la educación para enseñar al alumnado a desenvolverse en la era digital en la que vivimos, ofreciendo las herramientas y recursos necesarios que les permita hacer un buen uso de este fenómeno digital.

\section{REFERENCIAS}

Borrego, M., Guardeño, C., Jiménez, C., Montero, R., Negro, C., y Matas, A. (2019). Cuestionario Sobre Big Data en Educación. Escala de Valoración Del Big Data Aplicado a la Educación (VABIDAE). https://bit.ly/2mIdTIF.

Cuesta, F.T. (2019). La Inspección de Educación mirando al futuro Education Inspection looking at the future. Revista de Educación, (384), 123-146.

Camargo-Vega, J.J., Camargo-Ortega, J.F., y Joyanes-Aguilar, L. (2015). Knowing the Big Data. Revista Facultad de Ingeniería, 24(38), 63-77.

Daniel, B.K. (Ed.). (2017). Big data and learning analytics in higher education: current theory and practice. Springer.

De la fuente, R., y Quijano, C. (2018). Privacidad en Big Data. Computerworld Red de conocimiento. https://bit. ly/2p6A1xc.

Domínguez, D. (2018). Big data, analítica del aprendizaje y educación basada en datos. Universidad Nacional de
Educación a Distancia (UNED), 1-19. http://dx.doi. org/10.2139/ssrn.3124369

Eynon, R. (2013). The rise of Big Data: what does it mean for education, technology, and media research? Learning, Media, and Technology, 38(3), 237-240. https://doi.org/10 $.1080 / 17439884.2013 .771783$

Ferguson, R. (2014, 26 March). Learning analytics don't just measure students' progress-They can shape it. The guardian.https://www.theguardian.com/education/2014/ mar/26/learning-analytics-student-progress

García, M., y Fuentes, A. (2016). Uso de Big Data en contextos educativos. En REDINE (Eds.), EDUNOVATIC 2016-I Congreso Virtual internacional de Educación, Innovación y TIC (pp. 687-689). REDINE. Red de Investigación e Innovación Educativa.

Gartner (2014). Gartner says the Internet of Things will transform the Data Center. https://iot.do/gartner-says-internetthings-will-transform-data-center-2014-03.

Gasevic, D., Dawson, S., y Jovanovic, J. (2016). Ethics and privacy as enablers of learning analytics. Journal of Learn- 
ing Analytics, 3(1), 1-4. http://dx.doi.org/10.18608/ jla.2016.31.1

Gómez, M. (2016, octubre 17). El Big Data como fuente de empleo. https://bit.ly/2mNzvDy.

González, A., y Maroto, A. (2017). Big data para la investigación lingüística y la educación bilingüe. International Journal for 21st Century Education, 1(4), 33-41.

Guthrie, D. (2013, 15 August). The coming Big Data education revolution. US News. https://www.usnews.com/opinion/ articles/2013/08/15/why-big-data-not-moocs-willrevolutionize-education

Hair Jr., J.F., Black, W.C., Babin, B.J., y Anderson, R.E. (2014). Multivariate data analysis. Pearson Education Limited.

Ho, A. (2017). Advancing educational research and student privacy in the "Big Data" era. National Academy of Education.

IDC (2012). Worldwide Big Data technology and services 2012 2016 forecast. Market Analysis.

Laney, D. (2001). 3D data management: Controlling data volume, velocity and variety. META group research note, 6(70), 1.

Li, K.C., Jiang, H., Yang, L.T., y Cuzzocrea, A. (Eds.). (2015). Big data: Algorithms, analytics, and applications. CRC Press.

Macfadyen, L.P. (2017). Overcoming barriers to educational analytics: how systems thinking and pragmatism can help. Educational Technology, 57(1), 31-39.

Manyika, J., Chui, M., Brown, B., Bughin, J., Dobbs, R., Roxburgh, C., y Hung Byers, A. (2011). Big Data: The Next Frontier for Innovation, Competition, and Productivity.https:// www.mckinsey.com/business-functions/mckinseydigital/our-insights/big-data-the-next-frontier-forinnovation

Matas Terrón, A., Leiva Olivencia, J.J., y Franco Caballero, P. (2020). Big Data Irruption in Education / Irrupción del Big Data en la Educación. Píxel-Bit. Revista de Medios y Educación, (57), 59-90. 10.12795/pixelbit.2020.i57.02

Mohanty, H., Bhuyan, P., y Chenthati, D. (Eds.). (2015). Big data: A primer (Vol. 11). Springer.

Naujokaitienè, J., Tamoliūnė, G., Volungevičienè, A., y Duart, J.M. (2020). Using Learning analyctics to engange students: Improving teaching practices through informed interactions. Journal of New Approaches in Educational Research, 9(2), 231-244. 10.7821/naer.2020.7.561
Paniagua, P., y Cubo, S. (2018). Evaluación de las actitudes del profesorado hacia la aplicación de técnicas de "Big Data" en educación. En J. Valverde (Ed.), Campus digitales en la educación superior (pp. 221-234). Universidad de Extremadura.

Pedraza, J., y Molina, J.M. (2017). El Big Data en el ámbito de los resultados docentes. En E. Said y F.J. Durán (Eds.), Educación, participación y escenarios digitales: Debates sobre la mediación digital en el siglo XXI (pp. 62-70). Editorial Comares.

Poulovassilis, A. (2016). Big Data and education. http://www. dcs.bbk.ac.uk/oldsite/research/techreps/2016/ bbkcs-16-01.pdf

Roberts, L.D., Howell, J.A., y Seaman, K. (2017). Give me a Customizable Dashboard: Personalized Learning Analytics Dashboards in Higher Education. Technology, Knowledge and Learning, 3(22), 317-333.

Sancho-Gil, J. M. (2020). Digital technology as a trigger for learning: promises and realities. Digital Education Review, (37), 195-207.

Yudatama, U., Nazief, B. A., Hidayanto, A. N., y Mishbah, M. (2017, October). Factors affecting awareness and attitude of IT governance implementation in the higher education institution: A literature review. In R. Drezewski, G. Chakraborty, S. Nazir, L. Septem, U. Raba'ah, A. Prasetyo, Y. Wihardi, A. Pranolo, E. Junaeti, S.-H. Horng, H. Seok, L. Hernandez (Eds.), 2017 3rd International Conference on Science in Information Technology (ICSITech) (pp. 588-592). IEEE. 